\title{
ON THE GAPS IN THE SPECTRUM OF THE HILL EQUATION*
}

By C. R. PUTXAM (Purdue Lniversity)

1. Let $f=f(t)$ be a real-valued, continuous, periodic function of period 1 , so that

$$
f(t) \sim \sum_{n=-\infty}^{\infty} c_{n} \exp (2 \pi \text { int }), \quad\left(c_{-n}=\bar{c}_{n}\right),
$$

and consider the Hill equation

$$
x^{\prime \prime}+(\lambda+f(t)) x=0, \quad\left(\lambda \text { real; }{ }^{\prime}=d / d t\right) .
$$

It is known that (if $f \neq \equiv 0$ ) there exists a sequence of closed intervals $I_{k}: \lambda_{k} \leq \lambda \leq \lambda^{k}$ (region of stability), where $\lambda_{k}<\lambda^{k}<\lambda_{k+1}$ and $k=1,2, \cdots$, with the property that (2) has some solution $x \neq \equiv 0$ which is bounded on $-\infty<t<\infty$ if and only if $\lambda$ belongs to the closed set $S=\sum I_{k}$; cf. [7], p. 14. The complementary set of $S$ consists of a halfline $-\infty<\lambda<\lambda_{1}$ and the sequence of open intervals $J_{k}: \lambda^{k}<\lambda<\lambda_{k+1}, k=1,2, \cdots$. In several recent papers, various lower bounds for the value $\lambda_{1}$, the least point of the set $S$, in terms of the Fourier coefficients $c_{n}$ of $f(t)$, have been obtained; [11], [5], [3]. The present note will be devoted to the problem of obtaining estimates (upper bounds) of the lengths $\lambda_{k+1}-\lambda^{k}$ of the "gaps" $J_{k}$ of the set $S$ in terms of these Fourier coefficients.

It follows from [4], p. 613 , that the length of every gap $J_{k}$ is surely not greater than

$$
\limsup _{t \rightarrow \infty} f(t)-\liminf _{t \rightarrow \infty} f(t) \leq 4 \sum_{n=1}^{\infty}\left|c_{n}\right| \text {. }
$$

In addition, asymptotic estimates, as $\lambda^{k} \rightarrow \infty$, for these gaps are known; [2]. In fact, since $f(t)$ is uniformly continuous on $0 \leq t<\infty$, the lengths $\lambda_{k+1}-\lambda^{k}$ of the intervals $J_{k}$ tend to zero as $\lambda_{k+1} \rightarrow \infty$; loc. cit., p. 850. Furthermore, additional regularity conditions on $f(t)$ result in more refined estimates. It should be pointed out here that the investigations of [2] related to singular boundary value problems ([8]) on the half-line $0 \leq t<\infty$ determined by (2) and a linear, homogeneous boundary condition at $t=0$, and were not confined to the special case that $f(t)$ be periodic.

Let $m(\lambda)$, for $-\infty<\lambda<\infty$, be defined to be the distance from $\lambda$ to the set $S$ considered above, so that

$$
m(\lambda)=\text { g.l.b. }|\lambda-\mu|, \quad \mu \text { in } S .
$$

It will be shown in section 2 below that $m(\lambda)$ satisfies the inequality

$$
m^{2}(\lambda) \leq 2 \sum_{n=1}^{\infty}\left|c_{n}\right|^{2}, \quad \text { provided } \quad \lambda \geq-c_{0} .
$$

As a consequence of (4) and (5), one readily sees that the lengths $\lambda_{k+1}-\lambda^{k}$ of the gaps $J_{k}$ satisfy

$$
\lambda_{k+1}-\lambda^{k} \leq 2\left(2 \sum_{n=1}^{\infty}\left|c_{n}\right|^{2}\right)^{1 / 2}, \quad \text { provided } \quad \frac{1}{2}\left(\lambda_{k+1}+\lambda^{k}\right) \geq-c_{0} .
$$

It will remain undecided whether (6) actually must hold for all gaps $J_{k}$, so that the first inequality of (6) would hold without the proviso of the second inequality. In any

*Received April 13, 1953. 
case, it is readily seen that the estimate of (6), when it applies, is an improvement over that of (3), namely $4 \sum_{n=1}^{\infty}\left|c_{n}\right|$.

In this connection, it should be pointed out that Kato [3], by an adaptation of a relation used by Wintner [11], has obtained the inequality

$$
\lambda_{1} \geq-c_{0}-\left(\frac{1}{8}\right) \sum_{n=1}^{\infty}\left|c_{n}\right|^{2}
$$

for the least point $\lambda_{1}$ of the set $S$. (Wintner had previously shown that $\lambda_{1} \geq-c_{0}-2$. $\sum_{n=1}^{\infty}\left|c_{n}\right|^{2}$.) Consequently, it is easily seen that the first inequality of (6) is surely valid for all gaps $J_{k}$ if, for instance, the inequality

$$
\left(\frac{1}{8}\right) \sum_{n=1}^{\infty}\left|c_{n}\right|^{2} \leq\left(2 \sum_{n=1}^{\infty}\left|c_{n}\right|^{2}\right)^{1 / 2}
$$

holds. (If one normalizes $f$ so that its mean value is zero, hence $c_{0}=0$, this last inequality is equivalent to $\left.\int_{0}^{1} f^{2} d t \leq 256\right)$.

Before proceeding to the proof of (5), it can be noted that the first inequality of (5) surely becomes false if the restriction $\lambda \geq-c_{0}$ is dropped. In fact, if $f(t) \equiv c_{0}$, so that (2) becomes the differential equation of the harmonic oscillator, then $\sum_{n=1}^{\infty}\left|c_{n}\right|^{2}=0$, and (5) yields the known result that $m(\lambda) \equiv 0$ for $\lambda \geq-c_{0}$. However, $m(\lambda)>0$ for $\lambda<-c_{0}$, since $S$ is the half-line $-c_{0} \leq \lambda<\infty$.

2. The proof of (5) will depend upon certain results obtained in [6]. Let $g_{1}(t), g_{2}(t), \cdots$, denote a sequence of functions possessing continuous second derivatives on $0 \leq t<\infty$, satisfying

$$
g_{n}(0)=g_{n}^{\prime}(0)=0,
$$

and such that $g_{n}(t) \rightarrow 0$ uniformly on every finite $t$-interval $[0, T]$. Then, if $g_{n}$ and $L\left(g_{n}\right)$ (where $L(x) \equiv x^{\prime \prime}+f x$ ) are of class $L^{2}[0, \infty)$, the inequality

$$
m^{2}(\lambda) \liminf _{n \rightarrow \infty} \int_{0}^{\infty} g_{n}^{2} d t \leq \liminf _{n \rightarrow \infty} \int_{0}^{\infty}\left(L\left(g_{n}\right)+\lambda g_{n}\right)^{2} d t
$$

holds. This follows readily by a method analogous to that given in [6], p. 580. (It is to be noted that the set $S$ considered above is identical with the invariant spectrum (Weyl [8], p. 251) associated with the differential equation (2); [9], [1]. Moreover, the investigations of [6] related to the Weyl theory of singular boundary value problems, alluded to in section 1.)

Next, let $\mu>0$, and let $g_{n}=y_{n} h$, where $h=\sin \left(\mu^{\frac{1}{2}} t\right)$ or $h=\cos \left(\mu^{\frac{1}{2}} t\right)$, and the $y_{n}=y_{n}(t)$ are functions possessing continuous second derivatives on $0 \leq t<\infty$. In addition, suppose that $y_{n}(0)=y_{n}^{\prime}(0)=0$, so that (7) certainly holds, and that $y_{n}$ and $L\left(y_{n}\right)$ belong to $L^{2}(0, \infty)$. Finally, suppose that the $y_{n}$ are such that the "lim inf" appearing on the left side of the inequality (8) can be replaced by "lim" for both $h=\sin \left(\mu^{\frac{1}{2}} t\right)$ and $h=\cos \left(\mu^{\frac{1}{3}} t\right)$.

It follows from (8) that

$$
m^{2}(\lambda) \lim _{n \rightarrow \infty} \int_{0}^{\infty} y_{n}^{2} h^{2} d t \leq \liminf _{n \rightarrow \infty} \int_{0}^{\infty}\left(\left[y_{n}^{\prime \prime}+(\lambda-\mu+f) y_{n}\right] h+2 y_{n}^{\prime} h^{\prime}\right)^{2} d t
$$

If now the $y_{n}$ satisfy

$$
\int_{0}^{\infty} y_{n}^{\prime 2} d t \rightarrow 0, \quad \int_{0}^{\infty} y_{n}^{\prime \prime 2} d t \rightarrow 0, \quad(n \rightarrow \infty),
$$


it is seen that

$$
m^{2}(\lambda) \lim _{n \rightarrow \infty} \int_{0}^{\infty} y_{n}^{2} h^{2} d t \leq \liminf _{n \rightarrow \infty} \int_{0}^{\infty}(\lambda-\mu+f)^{2} y_{n}^{2} h^{2} d t
$$

Since (9) holds for both functions $h$, addition of the two corresponding inequality relations yields, in view of the fact that $\lim \inf A+\lim \inf B \leq \lim \inf (A+B)$, the inequality

$$
m^{2}(\lambda) \lim _{n \rightarrow \infty} \int_{0}^{\infty} y_{n}^{2} d t \leq \liminf _{n \rightarrow \infty} \int_{0}^{\infty}(\lambda-\mu+f)^{2} y_{n}^{2} d t
$$

Let $T>0$ and define the function $Y_{T}(t)$ on $0 \leq t<\infty$ so that the graph of $Y_{T}(t)$ on $0 \leq t \leq T$ consists of three line segments joining, in order, the four points $(0,0)$, $\left(1, T^{-\frac{1}{3}}\right),\left(T-1, T^{-\frac{1}{2}}\right)$, and $(T, 0)$. On $T<t<\infty$, let $Y_{T}(t) \equiv 0$. It is clear that the corners of this function can be smoothed out so as to obtain a function $y_{T}(t)$ satisfying the conditions imposed upon the $y_{n}$ above. Furthermore, it is clear that if $y_{n}=y_{T_{n}}$, where $T=T_{n} \rightarrow \infty$ as $n \rightarrow \infty$, one can arrange that the functions $y_{n}$ be such as to make (10) imply

$$
m^{2}(\lambda) \leq \liminf _{S \rightarrow \infty} S^{-1} \int_{0}^{s}(\lambda-\mu+f)^{2} d t, \quad(\mu \geq 0) .
$$

(It is clear that the inequality $\mu \geq 0$ in (11), and not merely $\mu>0$, can be allowed.) Now suppose that $\lambda \geq-c_{0}$ and choose $\mu \geq 0$ so that $\lambda-\mu=-c_{0}$. Then (11), (1), and the Parseval relation yield

$$
m^{2}(\lambda) \leq \int_{0}^{1}\left(-c_{0}+f\right)^{2} d t=2 \sum_{n=1}^{\infty}\left|c_{n}\right|^{2},
$$

so that the relation (5) is now proved.

\section{REFERENCES}

1. P. Hartman and A. Wintner, On the location of spectra of wave equations, Am. J. Math. 71, 214-217 (1949).

2. P. Hartman and C. R. Putnam, The gaps in the essential spectra of wave equations, Ibid. 72, 848-862 (1950).

3. T. Kato, Note on the least eigenvalue of the Hill equation, Q. Appl. Math. 10, 292-294 (1952).

4. C. R. Putnam, The cluster spectra of bounded potentials, Am. J. Math. 71, 612-620 (1949).

5. C. R. Putnam, On the least eigenvalue of Hill's equation, Q. Appl. Math. 9, 310-314 (1951).

6. C. R. Putnam, On the unboundedness of the essential spectrum, Am. J. Math. 74, 578-586 (1952).

7. M. J. O. Strutt, Lamesche, Mathieusche und verwandt Funktionen in Physik und Technik, Berlin, 1932.

8. H. Weyl, Ueber gewöhnliche Differentialgleichungen mit Singularitäten und die zugehörigen Entwicklungen willkürlicher Funktionen, Math. Ann. 68, 222-269 (1910).

9. A. Wintner, Stability and spectrum in the wave mechanics of lattices. Phys. Rev. 72, 81-82 (1947).

10. A. Wintner. A criterion of oscillatory stability, Q. Appl. Math. 7, 115-119 (1949).

11. A. Wintner, On the non-existence of conjugate points, Am. J. Math. 73, 368-380 (1951). 